\title{
Capacidade funcional submáxima e força muscular respiratória entre idosas praticantes de hidroginástica e dança: um estudo comparativo
}

\section{Submaximal functional capacity and respiratory muscle strength among elderly practitioners of hydrogymnastics and dance: a comparative study}

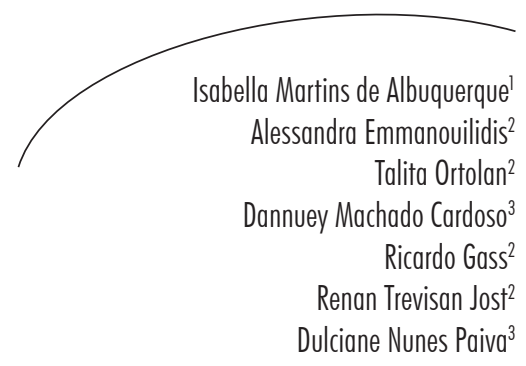

\section{Resumo}

O exercício físico é uma estratégia eficaz para prevenir e retardar as perdas funcionais do envelhecimento, mas há poucos estudos indicando qual a melhor modalidade para incrementar o status funcional do idoso. Objetivo: Comparar a força muscular respiratória (FMR) e a capacidade funcional submáxima de idosas praticantes de hidroginástica e dança. Material e Métodos: Estudo transversal com idosas do sexo feminino ( $\mathrm{n}=46$ ), praticantes de hidroginástica (Grupo Hidroginástica $-\mathrm{GH} ; \mathrm{n}=23$ ) e dança (Grupo Dança - GD; $\mathrm{n}=23$ ). Para medida da prática de atividade física, foi utilizado o International Physical Activit Questionnarie (IPAQ-versão curta); a FMR foi avaliada por meio da pressão inspiratória máxima (PImax) e da pressão expiratória máxima (PEmax), e a medida da capacidade funcional submáxima, realizada pelo Teste da Caminhada dos Seis Minutos (TC6m). Resultados: O GD apresentou maior PImax $\left(84 \pm 12,49 \mathrm{cmH}_{2} \mathrm{O}\right)$ quando comparado ao $\mathrm{GH}\left(63,35 \pm 10,47 \mathrm{cmH}_{2} \mathrm{O}\right)(\mathrm{p}<0,0001)$, e a PEmax não variou significativamente entre os grupos $(\mathrm{p}=0,08)$. Quanto à distância percorrida no TC6m, o GD $(616,53 \pm 60,81 \mathrm{~m})$ obteve melhor desempenho que o GH $(446,65 \pm 48,67 \mathrm{~m})(\mathrm{p}<0,0001)$. Quanto ao nível de atividade física, o GD apresentou 19 idosas $(82,61 \%)$ muito ativas e quatro $(17,39 \%)$ ativas, e o GH apresentou oito idosas $(30,76 \%)$ muito ativas e $15(69,23 \%)$ ativas. Conclusão: As idosas praticantes de dança evidenciaram maior força muscular inspiratória e capacidade funcional submáxima, possivelmente devido ao maior nível de atividade física e também pelo fato de a dança ser uma modalidade essencialmente aeróbia.

\footnotetext{
Departamento de Fisioterapia e Reabilitação. Universidade Federal de Santa Maria. Santa Maria, RS, Brasil.

2 Curso de Fisioterapia. Universidade de Santa Cruz do Sul. Santa Cruz do Sul, RS, Brasil.

Departamento de Educação Física e Saúde. Universidade de Santa Cruz do Sul. Santa Cruz do Sul, RS, Brasil.
}

Palavras- chave:

Envelhecimento. Atividade Motora. Exercício. Força Muscular. Terapia através da Dança. 


\section{Abstract}

Exercise is an effective strategy to prevent and slow the functional losses of aging, but there are few studies indicating the best method to improve functional status of the elderly. Objective: To compare respiratory muscle strength (RMS) and submaximal functional capacity of older practitioners of hydrogymnastics and dance. Material and Methods: A cross-sectional study with elderly women $(\mathrm{n}=46)$, practitioners of hydrogymnastics (Hydrogymnastics Group - HG; n=23) and dance (Dance Group - DG; $n=23$ ). For the measurement of physical activity, it was used the International Physical Activity Questionnaire (IPAQ - short version); the RMS was assessed by measurement of the maximal inspiratory pressure (MIP) and maximal expiratory pressure (MEP); and the measure of submaximal functional capacity was assessed by the six-minute walk test (6MWT). Results: The DG showed that higher MIP $(84 \pm 12.49$ $\left.\mathrm{cmH}_{2} \mathrm{O}\right)$ compared to the HG $\left(63.35 \pm 10.47 \mathrm{cmH}_{2} \mathrm{O}\right)(\mathrm{p}<0.0001)$ and the MEP did not differ significantly between the groups $(\mathrm{p}=0.08)$. The distance covered in the 6MWT DG $(616.53 \pm 60.81 \mathrm{~m})$ was better performed than HG $(446.65 \pm 48.67 \mathrm{~m})(\mathrm{p}<0.0001)$. For the physical activity level, the DG showed that 19 elderly women $(82.61 \%)$ were very active and four $(17.39 \%)$ active, and the HG showed that eight elderly women $(30.67 \%)$ were very active and 15 (69.23\%) active. Conclusion: The elderly women practitioners of dance showed higher respiratory muscle strength and submaximal functional capacity, possibly due to the higher level of physical activity and also because dance is primarily an aerobic modality.
Key words: Aging. Motor Activity. Exercise. Dance Therapy. Muscle Strength.

\section{INTRODUÇÃO}

O processo de envelhecimento apresenta como uma de suas consequências o declínio na força dos músculos esqueléticos e respiratórios, ${ }^{1}$ ocorrendo redução da força de recolhimento elástico dos pulmões e da complacência torácica, o que pode interferir na capacidade funcional e no desempenho das atividades de vida diária (AVDs) do idoso. ${ }^{2}$ A redução da massa e força muscular decorrentes do envelhecimento, ou sarcopenia, ocorre mesmo no idoso saudável e é considerada como o fator que contribui de forma mais significativa para a perda de independência e funcionalidade nessa faixa etária. ${ }^{3}$ Sabe-se que a participação regular, mesmo quando a prática é iniciada em uma fase tardia de vida, em programas de atividade física ou exercício físico, pode retardar esse declínio fisiológico. ${ }^{4-6}$ Estudo anterior indicou que o exercício aeróbio regular previne reduções na FMR relacionadas ao envelhecimento.
A dança é uma excelente forma de exercício físico aeróbico, e vários estudos ${ }^{8-10}$ vêm demonstrando a sua importância para a manutenção da força muscular, sustentação, equilíbrio e potência aeróbia na população idosa.

Baseada nas propriedades físicas da água, a hidroginástica vem sendo praticada com frequência em programas de exercício físico pela população idosa. ${ }^{11}$ Diversos estudos vêm demonstrando que os exercícios aquáticos promovem aumento da força muscular dos membros inferiores e aprimoramento do condicionamento cardiorrespiratório. ${ }^{12,13}$

Apesar dos benefícios potenciais, entretanto, são escassos os estudos que comparam os efeitos da prática da hidroginástica e da dança sobre a capacidade funcional e a força muscular respiratória. Sendo assim, o objetivo deste estudo foi comparar a distância percorrida no Teste da Caminhada de Seis Minutos e as pressões respiratórias estáticas de idosas praticantes de hidroginástica e dança. 


\section{MATERIAL E MÉTODOS}

\section{Participantes}

O presente estudo se caracteriza como transversal. Para tanto foram selecionadas, de forma intencional, voluntárias do sexo feminino e não-sedentárias, com idade entre 60 e 70 anos, que participavam do Projeto Ações para o Envelhecimento com Qualidade de Vida da Universidade de Santa Cruz do Sul (UNISC).

A amostra inicial foi composta por 52 idosas, e participaram do estudo 46. Como critério de inclusão as idosas que praticavam exercício físico por três vezes semanais com duração mínima de treino de 45 minutos e com assiduidade da prática por pelo menos um ano, sem histórico tabágico, com capacidade de deambulação sem assistência, isentas de patologias cardiorrespiratórias, neurológicas ou ortopédicas. Como critério de exclusão asidosas que apresentassem incapacidade de realizar corretamente os procedimentos, com instabilidade hemodinâmica ou presença de sintomas respiratórios. Foram excluídas seis, sendo duas por apresentarem histórico tabágico, uma por apresentar alteração na marcha durante o TC6m e três por terem idade acima de 70 anos.

As idosas foram então alocadas em dois grupos: Grupo Hidroginástica, vinculadas a um grupo praticante de exercícios físicos para idosos localizado na piscina pedagógica da universidade, e Grupo Dança, vinculadas a um grupo praticante de dança de salão intitulado "Grupo Arte de Viver", localizado no ginásio poliesportivo também da universidade.

A coleta de dados foi realizada no período de agosto a dezembro de 2010. O projeto foi submetido e aprovado pelo Comitê de Ética em Pesquisa da UNISC, sob o número de protocolo $n^{\circ}$ 2742/10 e, após, as participantes assinaram o Termo de Consentimento Livre e Esclarecido.

\section{Procedimentos}

Todos os procedimentos metodológicos foram realizados antes da prática do exercício físico habitual, divididos em duas etapas: a primeira compreendeu a avaliação da prática de atividade física, por meio da aplicação do International Physical Activity Questionnaire (IPAQ), versão reduzida, traduzido e validado no Brasil. ${ }^{14}$ Considerando os critérios de frequência, duração e tipo de atividade física, os indivíduos foram classificados em sedentários, insuficientemente ativos, ativos e muito ativos. Paralelamente à aplicação do IPAQ, foram verificadas as variáveis antropométricas e, na sequência, as voluntárias foram avaliadas quanto à FMR. A capacidade funcional submáxima, obtida por meio do Teste da Caminhada de Seis Minutos (TC6m), foi avaliada sete dias após a primeira etapa.

\section{Força muscular respiratória}

Para mensurar a FMR, utilizaram-se as medidas da pressão inspiratória máxima (PImax) e da pressão expiratória máxima (PEmax), avaliadas por meio de um manovacuômetro digital (MDI ${ }^{\circledR}$, MVD 300, GlobalMed. Brasil), seguindo as recomendações das Diretrizes dos Testes de Função Pulmonar. ${ }^{15} \mathrm{O}$ resultado da manovacuometria foi obtido após a realização de cinco manobras para cada valor desejado, obtendo-se três delas aceitáveis. Durante as manobras, não houve escape aéreo no bocal do aparelho e a duração de cada teste foi de pelo menos dois segundos. Os valores não diferiram entre si por mais de $10 \%$ do maior valor, onde se registrou a pressão mais elevada. ${ }^{15}$

\section{Capacidade funcional submáxima}

O TC6m foi realizado com monitorização prévia da pressão arterial sistólica (PAS), pressão arterial diastólica (PAD), frequência cardíaca (FC), frequência respiratória (FR) e saturação periférica de oxigênio $\left(\mathrm{SpO}_{2}\right)$, em um corredor sem obstáculos, reto, plano, com trinta metros de comprimento. As voluntárias foram instruídas previamente pelo avaliador, que utilizou estímulo verbal padronizado a cada minuto de caminhada, incentivado-as a percorrer a maior distância possível. A realização do teste seguiu o 
protocolo descrito pela American Thoracic Society, ${ }^{16}$ sendo que, ao seu final, as variáveis obtidas previamente foram mais uma vez mensuradas e as voluntárias foram questionadas quanto à percepção de dispnéia por meio da Escala de Borg modificada (escala 0-10).

\section{Grupo hidroginástica}

As aulas de hidroginástica foram ministradas três vezes por semana, com duração de 45 minutos. A piscina apresentava uma profundidade de $1,20 \mathrm{~m}$, medindo $25 \mathrm{~m} \times 12 \mathrm{~m}$, com água na temperatura aproximada de $30^{\circ}$ a $31^{\circ} \mathrm{C}$. As sessões consistiam em quatro fases: 1) aquecimento (alongamento e flexibilidade, método estático, durante cinco minutos); 2) exercícios aeróbicos (corridas, deslocamentos e movimentos combinados de braços e pernas, de modo intervalado, um minuto para atividade e um minuto para recuperação, durante 20 minutos); 3) exercícios localizados (força/resistência dos membros superiores, inferiores e abdominais, utilizando a resistência da água, durante 15 minutos); e 4) relaxamento (caminhadas lentas, por cinco minutos).

\section{Grupo dança}

As aulas de dança também foram ministradas três vezes por semana, com duração de 45 minutos. As sessões foram estruturadas em três fases: cinco minutos de alongamento e aquecimento inicial, 35 minutos de parte principal e cinco minutos de alongamento final e volta à calma. $\mathrm{O}$ alongamento consistiu em exercícios para os principais grupos musculares utilizados no dia a dia. Os ritmos de dança eram variados: merengue, xote, baião, vanera, mambo, foxtrot, valsa, rumba, swing, cha-cha e tango.

Análise dos dados

Com base nos dados obtidos no piloto do estudo, calculou-se que 20 indivíduos em cada grupo seriam necessários para determinar uma diferença de 10\% entre as médias da distância percorrida no TC6m entre os grupos, para um poder de $80 \%$ e alfa de $5 \%$.

Os resultados foram expressos em média e desvio-padrão ou mediana e intervalo interquartil. Foi utilizado o teste de Shapiro-Wilk para avaliar a normalidade da distribuição dos dados. As variáveis antropométricas, fisiológicas (PAS, PAD, FR, FC, $\mathrm{SpO}_{2}$, PImax e PEmax), escore da Escala de Borg e a distância percorrida predita no TC6m foram comparados entre os grupos por meio do teste $t$ Student para amostras independentes ou pelo teste U de Mann-Whitney, quando apropriado. $\mathrm{O}$ comportamento da distância percorrida e das variáveis fisiológicas foi comparado dentro de cada grupo pelo teste t Student pareado ou teste de Wilcoxon. Todas as análises foram feitas com auxílio do programa estatístico SPSS ${ }^{\circledR}$ for Windows ${ }^{\circledR}$ versão 14.0. Considerou-se o nível de significância estatística de $5 \%(p<0,05)$.

\section{RESULTADOS}

As 46 idosas participantes do estudo foram alocadas no $\mathrm{GH}(\mathrm{n}=23)$ e no GD $(\mathrm{n}=23)$ (figura 1). Não foram encontradas diferenças estatisticamente significativas entre os grupos nas variáveis antropométricas e fisiológicas (tabela 1). 


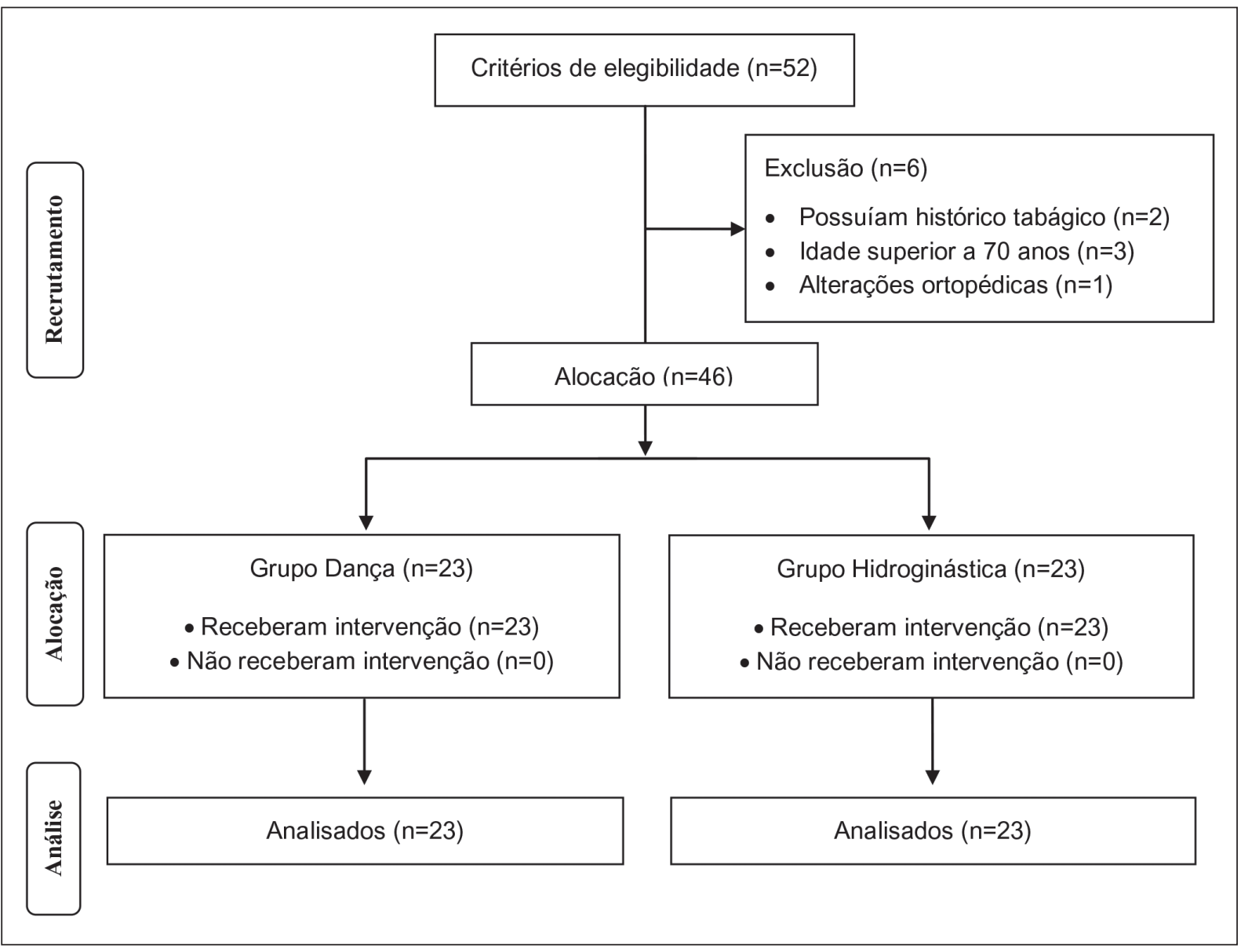

Figura 1 - Fluxograma da distribuição da amostra entre os grupos. Santa Cruz do Sul, RS, 2010.

Tabela 1 - Comparação das variáveis antropométricas e fisiológicas entre os grupos. Santa Cruz do Sul, RS, 2010.

\begin{tabular}{cccc}
\hline Variáveis & $\begin{array}{c}\text { Dança } \\
(n=23)\end{array}$ & $\begin{array}{c}\text { Hidroginástica } \\
(n=23)\end{array}$ & $p$ valor \\
\hline Idade $(\mathrm{anos})^{*}$ & $64,73(62-69)$ & $70,23(65,5-70)$ & 0,093 \\
IMC $\left(\mathrm{Kg} / \mathrm{m}^{2}\right)^{*}$ & $24,2(22,8-27,8)$ & $24,7(22,4-27,2)$ & 0,818 \\
FR (rpm) & $14,83 \pm 1,66$ & $15,34 \pm 1,67$ & 0,282 \\
FC (bpm) & $75,52 \pm 10,08$ & $75,69 \pm 9,54$ & 0,952 \\
PAS (mmHg)* & $120(120-130)$ & $120(120-132,5)$ & 0,323 \\
PAD (mmHg)* & $80(70-80)$ & $70(70-80)$ & 0,099 \\
SpO $(\%)$ & $97,52 \pm 0,94$ & $96,23 \pm 2,51$ & 0,091 \\
Borg (escore) & $0,04 \pm 0,14$ & $0,32 \pm 0,88$ & 0,418 \\
\hline
\end{tabular}

* Variáveis descritas em mediana e intervalo interquartil; demais variáveis com distribuição normal, em média \pm desvio-padrão. IMC: índice de massa corporal; FR: frequência respiratória; FC: frequência cardíaca; PAS: pressão arterial sistólica; PAD: pressão arterial diastólica; SpO saturação periférica de oxigênio. Significância estatística: $\mathrm{p}<0,05$. 
Em relação ao nível de atividade física, segundo o questionário IPAQ, no GD, 19 idosas $(82,61 \%)$ foram consideradas muito ativas e quatro $(17,39 \%)$ foram consideradas ativas. Já no $\mathrm{GH}$, oito idosas $(30,76 \%)$ foram consideradas muito ativas e $15(69,23 \%)$ foram consideradas ativas.
A PImax foi significativamente maior no GD (84 $\left.\pm 12,49 \mathrm{cmH}_{2} \mathrm{O}\right)$, quando comparada ao $\mathrm{GH}\left(63,35 \pm 10,47 \mathrm{cmH}_{2} \mathrm{O}\right)(\mathrm{p}<0,0001)$. Ao analisarmos os valores de PEmax, não houve diferença estatisticamente significativa $(\mathrm{p}=0,08)$ entre os Grupos Dança $\left(101,41 \pm 15,15 \mathrm{cmH}_{2} \mathrm{O}\right)$ e Hidroginástica $\left(90,54 \pm 14,78 \mathrm{cmH}_{2} \mathrm{O}\right)$ (figura 2).

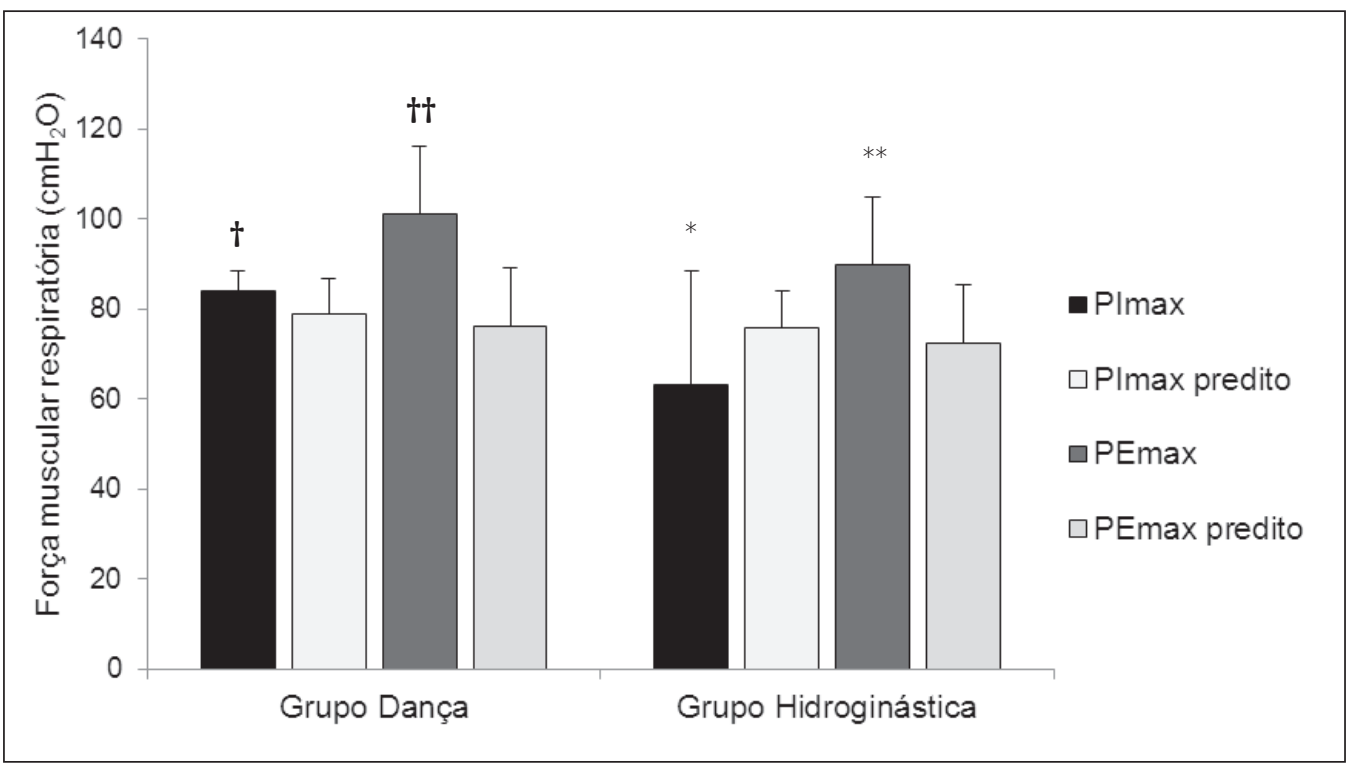

†Comparação PImax entre dança e hidroginástica, $p<0,0001 ;$ ††Comparação entre PEmax observada e predito no grupo dança $(p<0,0001)$; *Comparação entre PImax observada e predito no grupo hidroginástica $(\mathrm{p}=0,009) ;{ }^{* *}$ Comparação entre PEmax observada e predito no grupo hidroginástica $(\mathrm{p}=0,058)$.

Figura 2 - Comparação dos valores da Pressão Inspiratória Máxima (PImax) e Pressão Expiratória Máxima (PEmax) entre os grupos e seu respectivo valor predito. Santa Cruz do Sul, RS, 2010.

É importante ressaltar que o GD evidenciou valores significativos maiores de PImax em relação ao predito $\left(78,86 \pm 7,37 \mathrm{cmH}_{2} \mathrm{O} ; \mathrm{p}=\right.$ 0,062), bem como da PEmax (76,34 \pm 6,96 $\left.\mathrm{cmH}_{2} \mathrm{O} ; \mathrm{p}<0,0001\right)$. No GH, a PImax não se diferenciou em relação ao predito $(75,99 \pm$ $\left.12 \mathrm{cmH}_{2} \mathrm{O} ; \mathrm{p}=0,009\right)$; entretanto, a PEmax apresentou-se significativamente maior em relação ao predito $\left(72,38 \pm 13,45 \mathrm{cmH}_{2} \mathrm{O} ; \mathrm{p}=\right.$ 0,001 ) (figura 2).
Com relação à distância percorrida no TC6m, o GD (616,53 \pm 60,81 m) obteve um desempenho significativamente maior $(\mathrm{p}<0,0001)$, quando comparado ao GH $(446,65 \pm 48,67 \mathrm{~m})$. Ao compararmos os valores preditos de normalidade, segundo a equação de referência de Enright ${ }^{17}$ no TC6m (485,24 $\pm 39,72 \mathrm{~m})$ no GD, observamos que este grupo obteve desempenho significativamente maior na distância percorrida, representando um valor de $27 \%$ a mais que o predito. Este mesmo desempenho, entretanto, não se repetiu no $\mathrm{GH}$ $(452,72 \pm 36,29 \mathrm{~m})$ (figura 3). 


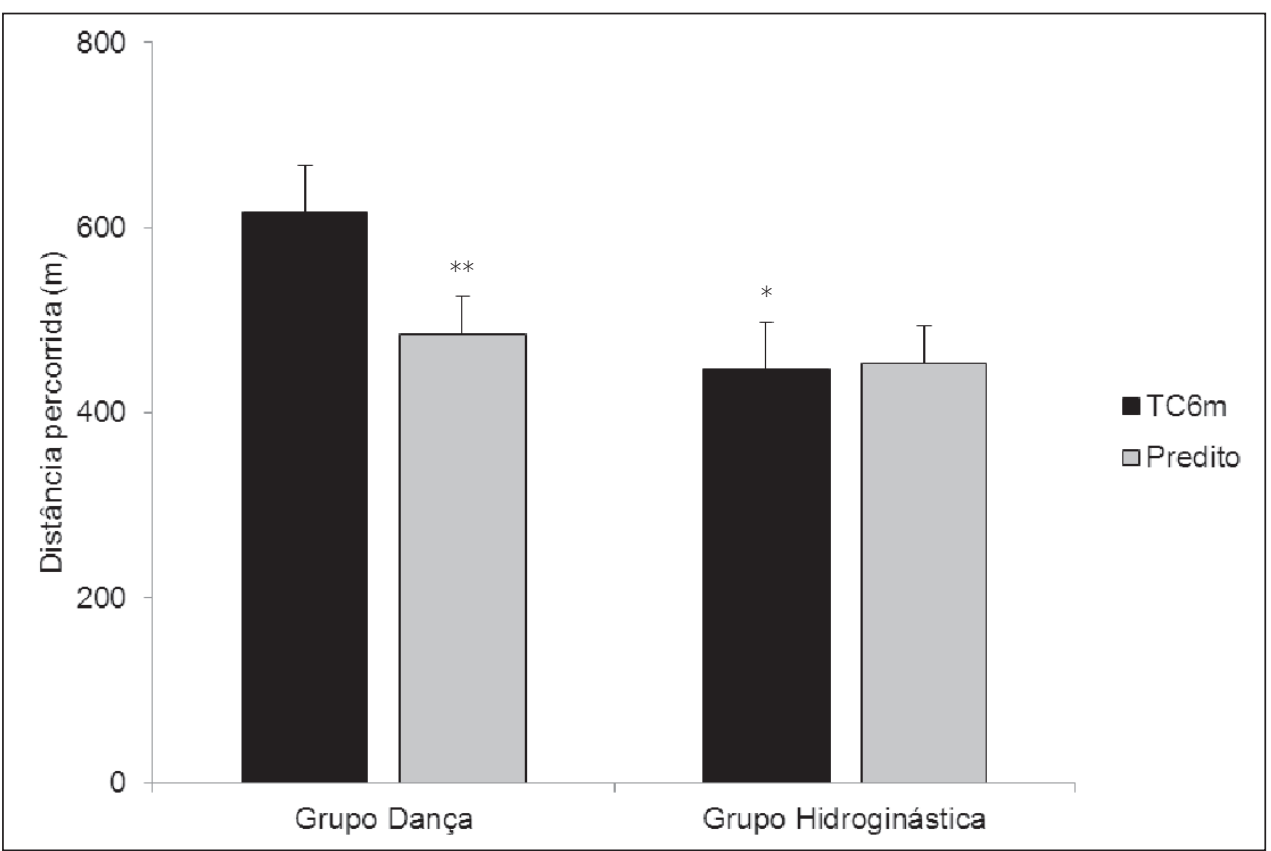

*Comparação entre os valores da distância percorrida no TC6m dança e hidroginástica ( $<<0,0001)$; **Comparação entre os valores da distância percorrida no TC6m obtidos e preditos no grupo dança $(\mathrm{p}<0,0001)$.

Figura 3 - Comparação dos valores da distância percorrida no TC6m e o predito na literatura entre os grupos. Santa Cruz do Sul, RS, 2010.

\section{DISCUSSÃO}

Este estudo objetivou comparar a capacidade funcional submáxima eaFMR deidosas praticantes de hidroginástica e dança. Os resultados mostram que as idosas que praticam dança obtiveram maior força muscular inspiratória e percorreram maior distância no TC6m, quando comparadas às praticantes de hidroginástica.

A literatura vem demonstrando que a dança é uma forma benéfica de atividade física para idosos, havendo benefícios físicos principalmente no que tange à melhora do equilíbrio, o que reduz o risco de quedas. ${ }^{18}$ Murrock \& Gary ${ }^{19}$ concluíram em seu estudo com 126 indivíduos, com faixa etária de 36 a 82 anos, que a dança aumenta significativamente a capacidade funcional de mulheres afroamericanas. Sendo assim, trata-se de uma forma de atividade física benéfica tanto para adultos jovens quanto para idosos.
Os exercícios aquáticos, por sua vez, têm sido utilizados como forma de terapia e atividade física em pacientes com doença de Parkinson, ${ }^{20}$ osteoartrite $^{21}$ e também em idosos saudáveis. ${ }^{22}$ A hidroginástica, utilizando as características físicas da água como viscosidade, flutuabilidade, resistência e empuxo, pode proporcionar ao idoso maior segurança no exercício. ${ }^{11,23}$ Tsourlou et al. ${ }^{24}$ realizaram um programa de treinamento aquático de 24 semanas e concluíram que o método proporcionou melhora significativa na aptidão neuromuscular e funcional em mulheres idosas saudáveis. Contudo, não há estudos que comparem essas duas formas de exercício físico quanto ao incremento da FMR e da capacidade funcional submáxima em idosas.

No GD, ao se comparar a FMR e a distância percorrida no TC6m, em relação ao seu valor predito, constatou-se aumento significativo de tais valores. Estudos indicam que a dança é capaz de aumentar significativamente a resistência 
muscular e a capacidade aeróbica de idosos, $, 2,25$ o que pode refletir no aumento da força dos músculos respiratórios. Além disso, Grant et al. ${ }^{26}$ relataram que a dança é capaz de melhorar o condicionamento aeróbico avaliado por meio do teste de esforço cardiopulmonar em homens e mulheres acima de 50 anos.

No grupo GH, entretanto, essa diferença não foi observada em todas as variáveis, onde apenas a PEmax foi significativamente maior em relação a seu valor predito. O recrutamento dos músculos abdominais durante as atividades nãorespiratórias, como a simples imersão na água, por exemplo, pode ter sido a fonte do estímulo de treinamento de força sobre o diafragma e músculos expiratórios. Ao realizar manobras que aumentam a pressão intra-abdominal, o diafragma é ativado para minimizar a transmissão de alta pressão intra-abominal ao tórax. ${ }^{27,28}$ Contraditoriamente aos resultados encontrados no presente estudo, onde houve variação significativa apenas da PEmax, Summerhill et al. encontraram melhora tanto da PImax quanto da PEmax de idosos. Segundo os mesmos autores, os idosos ativos rotineiramente envolvidos em atividades vigorosas que elevavam a pressão intra-abdominal tendem a fortalecer tanto o diafragma quanto a musculatura abdominal.

Ao analisar a comparação entre os grupos, pode-se observar que o GD apresentou valores de FMR e de capacidade funcional submáxima maiores que o $\mathrm{GH}$, tanto no que se refere à PImax, quanto à distância percorrida no TC6m. Outro fator causal possível é o maior nível de atividade física no GD. Pires et al. ${ }^{29}$ demonstraram, em 122 indivíduos com idade entre 18 e 80 anos, que quanto maior a idade, menor é a distância percorrida no Teste de Caminhada. Fernandes et al..$^{30}$ colocam que o nível de atividade física interfere tanto no nível cognitivo quanto na distância percorrida no TC6m.

Não obstante, sabe-se que os protocolos de hidroginástica podem apresentar variabilidade em seus componentes, ${ }^{31,32}$ o que pode interferir no incremento de força e capacidade funcional submáxima por não serem atividades aeróbicas. Diferentemente, a dança caracteriza-se como uma atividade exclusivamente aeróbica, o que também pode explicar o melhor desempenho do GD nas variáveis analisadas. Recente revisão sistemática demonstrou que há forte evidência de efeitos positivos da prática de dança, sobretudo em idosas, quanto a potência aeróbia, resistência muscular de membros inferiores, força, flexibilidade e velocidade da marcha. ${ }^{33}$

É importante mencionar que a não quantificação do tempo de atividade física e a falta de avaliação do nível cognitivo foram possíveis limitações do presente estudo. Ressalta-se que, pela menor prática de atividade física do $\mathrm{GH}$, o nível cognitivo pode ter sido um fator limitante para as idosas não apresentarem superioridade, em relação aos seus valores preditos, nos desfechos "força muscular inspiratória' e "capacidade funcional submáxima".

\section{CONCLUSÃO}

Considerando as limitações mencionadas, os resultados indicam que o Grupo Dança apresentou maior força muscular respiratória e capacidade funcional submáxima quando comparado ao Grupo Hidroginástica, possivelmente devido ao maior nível de atividade física e também pelo fato de a dança ser uma modalidade que incrementa a potência aeróbia, a resistência muscular de membros inferiores, a força, a flexibilidade e a velocidade da marcha em idosos.

Aconselham-se iniciativas para o aumento da prática de exercícios e atividades físicas regulares para indivíduos idosos, a fim de atenuar o declínio da aptidão cardiorrespiratória. Sugerese ainda que a otimização dessas funções deva ser incluída nos programas de promoção em saúde e prevenção de doenças, com o propósito de manter e/ou melhorar a capacidade funcional, o que poderia minimizar o impacto do processo de envelhecimento. 


\section{REFERÊNCIAS}

1. Tolep K, Higgins N, Muza S, Criner G, Kelsen SG. Comparison of diaphragm strength between healthy adult elderly and young men. Am J Respir Crit Care Med 1995;152(2):677-82.

2. Doherty TJ. Invited review: Aging and sarcopenia. J Appl Physiol 2003;95(4):1717-27.

3. Malafarina V, Uriz-Otano F, Iniesta R, GilGuerrero L. Sarcopenia in the elderly: diagnosis, physiopathology and treatment. Maturitas 2012;71(2):109-14.

4. Nelson ME, Rejeski WJ, Blair SN, Duncan PW, Judge JO, King AC, et al. Physical activity and public health in older adults: recommendation from the American College of Sports Medicine and the American Heart Association. Med Sci Sports Exerc 2007;39(8):1435-45.

5. Chodzko-Zajko WJ, Proctor DN, Fiatarone Singh MA, Minson CT, Nigg CR, Salem GJ, et al. American College of Sports Medicine position stand. Exercise and physical activity for older adults. Med Sci Sports Exerc 2009;41(7):1510-30.

6. King AC, Guralnik JM. Maximizing the potential of an aging population. JAMA 2010;304(17):1944-5.

7. Summerhill EM, Angov N, Garber C, McCool FD. Respiratory muscle strength in the physically active elderly. Lung 2007;185(6):315-20.

8. Eyigor S, Karapolat H, Durmaz B, Ibisoglu U, Cakir S. A randomized controlled trial of Turkish folklore dance on the physical performance, balance, depression and quality of life in older women. Arch Gerontol Geriatr 2009;48(1):84-8.

9. Lima MMS, Vieira AP. Ballroom dance as therapy for the elderly in Brazil. Am J Dan Ther 2007;29(2):129-42.

10. Stacey G, Stickley T. Dancing to keep young at heart. Ment Health Pract 2008;11:34-8.

11. Katsura Y, Yoshikawa T, Ueda SY, Usui T, Sotobayashi D, Nakao H, et al. Effects of aquatic exercise training using water-resistance equipment in elderly. Eur J Appl Physiol 2010;108(5):957-64.

12. Gusi N, Tomas-Carus P, Hakkinen A, Hakkinen K, Ortega-Alonso A. Exercise in waist-high warm water decreases pain and improves health-related quality of life and strength in the lower extremities in women with fibromyalgia. Arthritis Rheum 2006;55(1):66-73.

13. Wang TJ, Belza B, Elaine'Thompson F, Whitney JD, Bennett K. Effects of aquatic exercise on flexibility, strength and aerobic fitness in adults with osteoarthritis of the hip or knee. J Adv Nurs 2007;57(2):141-52.
14. Matsudo S, Araújo T, Marsudo V, Andrade D, Andrade E, Oliveira LC, et al. Questionário internacional de atividade física (IPAQ): estudo de validade e reprodutibilidade no Brasil. Rev Bras Ativ Fis Saúde 2001;6(2):5-18.

15. Pereira CAC. Projeto Diretrizes: Testes de função pulmonar. Sociedade Brasileira de Pneumologia e Tisiologia. L Pneumol 2001;2:155-62.

16. ATC Committee on Proficiency Standards for Clinical Pulmonary Function Laboratories. Laboratories ATSCoPSfCPF. ATS statement: guidelines for the six-minute walk test. Am J Respir Crit Care Med 2002;166(1):111-7.

17. Enright PL, Sherrill DL. Reference equations for the six-minute walk in healthy adults. Am J Respir Crit Care Med 1998;158(5 Pt 1):1384-7.

18. Judge JO. Balance training to maintain mobility and prevent disability. Am J Prev Med 2003;25(3 Suppl 2):150-6.

19. Murrock CJ, Gary FA. A culturally-specific dance intervention to increase functional capacity in African American women. J Cult Divers 2008;15(4):168-73.

20. Vivas J, Arias P, Cudeiro J. Aquatic therapy versus conventional land-based therapy for Parkinson's disease: an open-label pilot study. Arch Phys Med Rehabil 2011;92(8):1202-10.

21. Wang TJ, Lee SC, Liang SY, Tung HH, Wu SF, Lin YP. Comparing the efficacy of aquatic exercises and land-based exercises for patients with knee osteoarthritis. J Clin Nurs 2011;20(17-18):2609-22.

22. Avelar NC, Bastone AC, Alcântara MA, Gomes WF. Effectiveness of aquatic and non-aquatic lower limb muscle endurance training in the static and dynamic balance of elderly people. Rev Bras Fisioter 2010;14(3):229-36.

23. Suomi R, Koceja DM. Postural sway characteristics in women with lower extremity arthritis before and after an aquatic exercise intervention. Arch Phys Med Rehabil 2000;81(6):780-5.

24. Tsourlou T, Benik A, Dipla K, Zafeiridis A, Kellis $\mathrm{S}$. The effects of a twenty-four-week aquatic training program on muscular strength performance in healthy elderly women. J Strength Cond Res 2006;20(4):811-8.

25. McKinley P, Jacobson A, Leroux A, Bednarczyk V, Rossignol M, Fung J. Effect of a community-based Argentine tango dance program on functional balance and confidence in older adults. J Aging Phys Act. 2008;16(4):435-53. 
26. Grant S, Corbett K, Todd K, Davies C, Aitchison T, Mutrie N, et al. A comparison of physiological responses and rating of perceived exertion in two modes of aerobic exercise in men and women over 50 years of age. Br J Sports Med 2002;36(4):276-80; discussion 281 .

27. Al-Bilbeisi F, McCool FD. Diaphragm recruitment during nonrespiratory activities. Am J Respir Crit Care Med 2000;162(2 Pt 1):456-9.

28. DePalo VA, Parker AL, Al-Bilbeisi F, Mc Cool FD. Respiratory muscle strength training with nonrespiratory maneuvers. J Appl Physiol 2004;96(2):731-4.

29. Pires SR, Oliveira AC, Parreira VF, Britto RR. Teste de caminhada de seis minutos em diferentes faixas etárias e índices de massa corporal. Rev Bras Fisiot 2007;11(2):147-51.
30. Fernandes D, Cardoso DM, Jost RT, Paiva DN, Albuquerque IM. Correlação entre capacidade funcional e nível cognitivo em idosas hígidas sedentárias e ativas. Ciência em Movimento 2011;13:47-54.

31. Paiva DN, Jost RT, Schuster J, Cardoso DM, Albuquerque IM. Efeito da fisioterapia aquática sobre a função pulmonar e a qualidade de vida em portadoras de câncer de mama. FIEP Bulletin 2012;82:585-8

32. Garcia C, Barela JA, Viana AR, Barela AM. Influence of gymnastics training on the development of postural control. Neurosci Lett 2011;492(1):29-32.

33. Keogh JW, Kilding A, Pidgeon P, Ashley L, Gillis D. Physical benefits of dancing for healthy older adults: a review. J Aging Phys Act 2009;17(4):479-500. 